\title{
Efficient Terahertz Pulse Generation in Laser-Induced Gas Plasmas
}

\author{
T. LÖFfler*, M. Kress, M. Thomson and H.G. Roskos \\ Physikalisches Institut, Johann Wolfgang Goethe-Universität Frankfurt \\ Robert-Mayer-Str. 2-4, 60054 Frankfurt (Main), Germany
}

\begin{abstract}
In this contribution, we review the development of sources for far-infrared (terahertz) radiation based on laser-generated gas plasmas. We describe several generation mechanisms based on ponderomotive forces, external field screening, and optical second-harmonic biasing. These methods are compared with the standard techniques with respect to the achievable terahertz pulse energy.
\end{abstract}

PACS numbers: 42.65.Re, 72.30.+q, 78.47.+p

\section{Introduction}

With state-of-the-art amplifier laser systems one easily reaches pulse energies sufficient to ionize atoms and molecules in focused beams. If this is done in a gaseous medium at ambient pressure (e.g., in air), a plasma with a length of a few hundred $\mu \mathrm{m}$ and a diameter of up to $100 \mu \mathrm{m}$ can be produced by a $1 \mathrm{kHz}$ $150 \mathrm{fs}$ Ti:sapphire laser system with laser pulse energies in the $500 \mu \mathrm{J}$ regime. The plasma formation process is strongly influenced by the optical non-linearity of the plasma itself which leads to strong defocusing of the beam and a limitation of the maximum plasma density to a few $10 \%$ of the particle density [1]. A CCD image of a laser-induced plasma is shown in Fig. 1.

Terahertz $(\mathrm{THz})$ emission from laser-induced plasmas was found for the first time by Hamster et al. in 1993 [2-4]. Their experiment was based on the acceleration of the charged electrons and ions by the ponderomotive forces exerted by the intense light pulses. Recently, we demonstrated that the emission process can be enhanced and steered to the forward direction by applying a static bias field to the plasma [5]. In following reports, Cook et al. showed that even more efficient

*corresponding author; e-mail: t.loeffler@physik.uni-frankfurt.de 


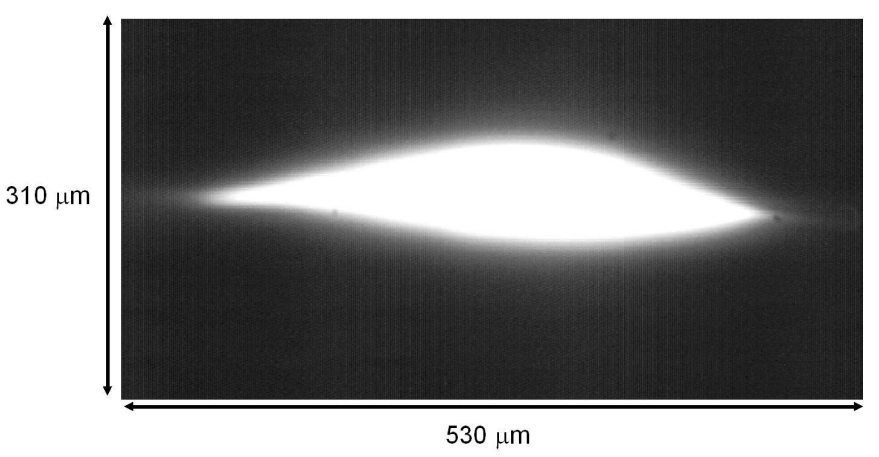

Fig. 1. CCD image of a laser-induced gas plasma. The laser pulse had an energy of $550 \mu \mathrm{J}$, a pulse length of $150 \mathrm{fs}$, and it was focused with a lens of $40 \mathrm{~mm}$ focal length. The laser pulse propagated from right to left. The asymmetry of the plasma volume relative to the beam axis reflects an asymmetric pulse shape.

THz-pulse generation is achieved when laser pulses composed of a superposition of both fundamental and second-harmonic spectral components are focused into air $[6,7]$. We demonstrated that this way of $\mathrm{THz}$ generation is clearly related to the formation of a plasma in the focus $[8,9]$.

\section{Experimental arrangement}

In our experiments, we employ a $1 \mathrm{kHz}$ Ti:sapphire laser system (Clark CPA 2001), which provides $150 \mathrm{fs}$ laser pulses at $775 \mathrm{~nm}$ at a maximum pulse energy of $860 \mu \mathrm{J}$. We work with various setups optimized for the respective $\mathrm{THz}$ generation mechanism (see Fig. 2). (i) For measurements of signals resulting from ponderomotive forces, the fact that $\mathrm{THz}$ emission does not occur in forward direction is important. The $\mathrm{THz}$ signal is collected sideways from the beam axis. (ii) In measurements with electrically biased plasmas, two electrodes with round tips apply an external field to the plasma and the $\mathrm{THz}$ signal emitted in forward direction is detected. (iii) Following the second-harmonic-biasing approach of Cook et al. [6, 7], laser pulses are focused into a $100 \mu \mathrm{m}$ thick $\beta$-barium-borate (BBO) crystal type-I phase-matched for second-harmonic generation (SHG). The efficiency of SHG is controlled by variation of the distance of the BBO crystal from the beam waist where the $\mathrm{THz}$ radiation is generated in air. Because of the different phase velocities of the $\omega$ - and $2 \omega$-fields in air, the relative phase between the fundamental and the second-harmonic components at the focus depends on the distance between BBO crystal and focus. The measurements described in the following are performed with the relative phase optimized for maximum $\mathrm{THz}$ -generation efficiency which is achieved by tilting a glass platelet in the beam path until maximum $\mathrm{THz}$ power is obtained. (iv) A standard large-aperture GaAs emitter serves as a reference emitter. It consists of a semi-insulating GaAs wafer 


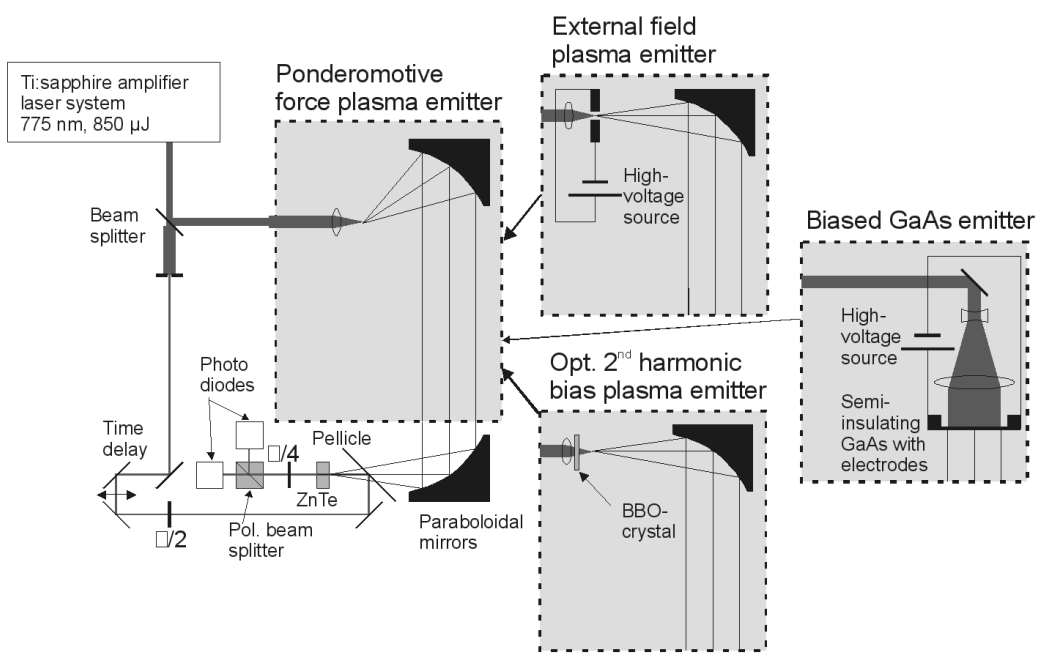

Fig. 2. Experimental arrangement for various emitter types.

with metal electrodes defining an active emitter area of $3 \mathrm{~cm} \times 3 \mathrm{~cm}$ size [10]. The static bias is $1 \mathrm{kV} / \mathrm{cm}$. Illumination occurs with the help of a telescope.

In the case of all three plasma sources, the emitted $\mathrm{THz}$ radiation is collimated by an off-axis paraboloid $(f=50 \mathrm{~mm})$ and then focused with a second one $(f=50 \mathrm{~mm})$ onto a $1 \mathrm{~mm}$ thick ZnTe crystal for electro-optical detection. In the case of the semiconductor emitter, the first paraboloid is left out because the emission from the large-area-illuminated wafer occurs in a collimated manner anyway.

\section{3. $\mathrm{THz}$ emission based on ponderomotive forces}

Ponderomotive forces result from the fact that a charged particle within an oscillating electric field will perform an oscillatory motion which brings the particle into regions of different field intensity. As the particle acceleration is weaker in regions of low field amplitude, the particle will gradually propagate out of the high-intensity regions into the low-intensity ones. The related force is called the ponderomotive force and is described by [4]:

$$
\boldsymbol{F}_{\text {pon }}=-\nabla \frac{q^{2} I_{\mathrm{opt}}}{2 \varepsilon_{0} c_{0} m_{q} \omega_{\mathrm{opt}}^{2}} .
$$

Here $q$ and $m_{q}$ denote the charge and the mass of the particle. $I_{\mathrm{opt}}$ and $\omega_{\mathrm{opt}}$ are the intensity and the frequency of the light. Within the focus of a laser beam from a pulsed amplifier laser, the ponderomotive force is large enough to modify the spatial distributions of the electrons and ions significantly on an ultrafast time scale. Due to the lower mass of the electrons they are accelerated out of the focal region much more rapidly than the ions. This leads to the formation of a 
rotationally symmetric polarization in the focus which has no net dipole moment and hence is not expected to emit $\mathrm{THz}$ radiation in forward direction but still is the source of radiation emitted non-collinearly with the pump light as was first shown theoretically and experimentally by Hamster et al. [2-4].

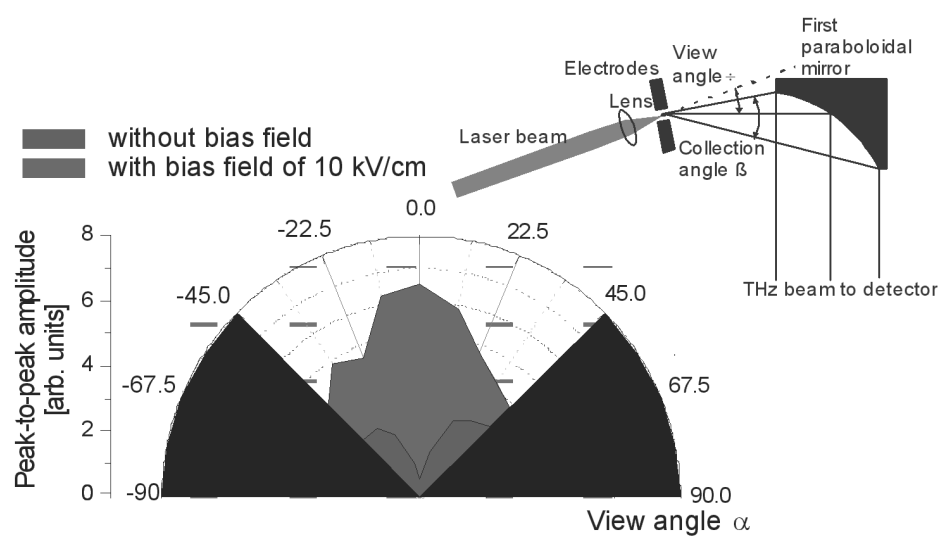

Fig. 3. Measurement of the radial emission pattern of the $\mathrm{THz}$ radiation based on the ponderomotive forces (light grey) and based on an applied external field (dark grey).

Emission occurs into a cone around the beam axis. An experimental verification by us is shown in Fig. 3. THz radiation emitted from the plasma is collected with the help of a paraboloidal mirror whose optical axis is gradually rotated away from the optical beam axis allowing us to map out the radial characteristics of the emission pattern. The view angle (radial angle) is changed from $-45^{\circ}$ to $45^{\circ}$. The ensuing radiation pattern is displayed in Fig. 3 in light grey color. As expected, the emission intensity increases with the radial angle, and the radiation vanishes in forward direction. For comparison, Fig. 3 also displays in dark grey the emission pattern obtained with an electrically biased plasma (see below). The forward orientation of the emission for this case is clearly visible.

The THz-generation process based on ponderomotive forces has a rather low conversion efficiency (see Sec. 5), although one expects very efficient emission for ultrashort laser pulses with sub-10 fs duration [11].

\section{THz emission based on external field screening}

One approach to increase the efficiency and to steer the emission into the forward direction is to apply a static bias field to the plasma. In this case, $\mathrm{THz}$ generation is associated with ultrafast screening of the external field within the plasma, and the corresponding displacement current is the source of an intense $\mathrm{THz}$ pulse [5]. Figure 4 displays $\mathrm{THz}$ field transients for various bias fields (left part) and the pulse spectrum for the maximum bias field (right part). The pulse 


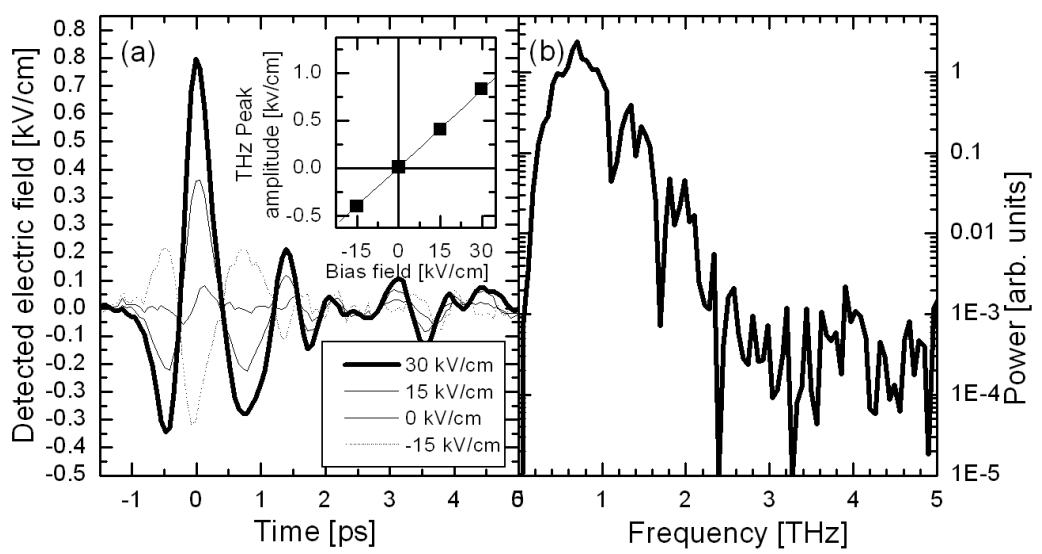

Fig. 4. Left part: detected electric $\mathrm{THz}$ field from an electrically biased plasma as a function of time for various bias fields. Inset: field amplitude as a function of bias field. Right part: power spectrum of the $\mathrm{THz}$ pulse obtained at a static bias field of $30 \mathrm{kV} / \mathrm{cm}$.

amplitude (energy) of the $\mathrm{THz}$ pulses scales linearly (quadratically) with the external bias field (see inset of Fig. 4) as for most opto-electronic emitters. The maximal achievable $\mathrm{THz}$ pulse energy for a given laser pulse energy is limited by the maximal external bias field which can be applied without dielectric breakdown of the gas. We have demonstrated that the breakdown field and, with it, the maximal $\mathrm{THz}$ pulse energy can be enhanced significantly if the gas pressure is raised [12].

\section{THz emission based on optical second-harmonic biasing}

The efficiency of an emitter with static bias is still limited by the extremely fast screening of the bias field which requires displacement of the ions and electrons by only a fraction of an atomic radius. Higher efficiency requires application of an $\mathrm{AC}$ bias at a frequency above the plasma frequency. Cook et al. introduced an elegant realization of this concept. They showed experimentally that efficient $\mathrm{THz}$ pulse generation is achieved when laser pulses composed of a superposition of both fundamental and second-harmonic (SH) spectral components are focused into air $[6,7]$. Here, the $\mathrm{SH}$ radiation takes the role of an $\mathrm{AC}$ bias field with a very high frequency. One should point out that this view - although being very intuitive - only grasps part of the real operational concept because the coherence between fundamental and $\mathrm{SH}$ waves plays an important role for the THz-generation process. In fact, a non-linear-optical description is more appropriate and was employed by Cook et al. in their papers.

Some of our own experimental results with this approach are shown in Fig. 5. The data are corrected for additional $\mathrm{THz}$ signal components which are generated in the BBO crystal itself $[8,9]$. Only THz-signal components are displayed which 


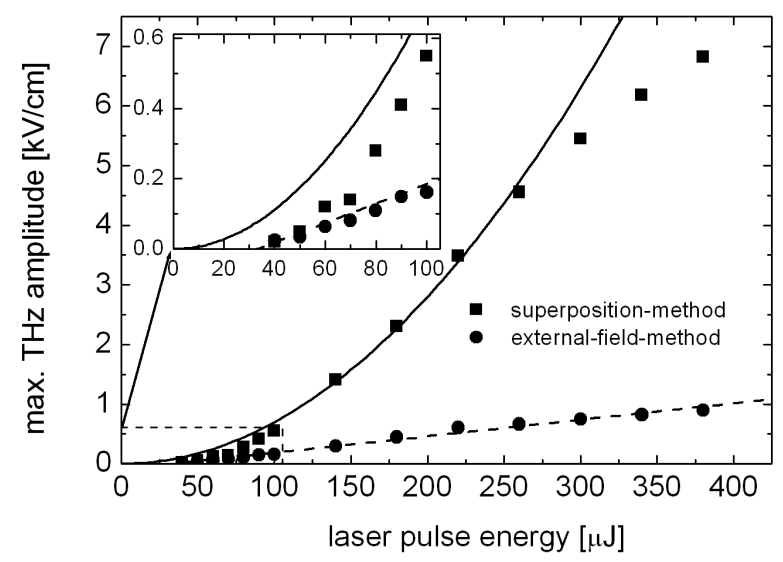

Fig. 5. Amplitude of the measured $\mathrm{THz}$ transients as a function of optical pulse energy for the external-field method (dots) and for the superposition of the fundamental wave with its second harmonic wave (squares). Inset: magnification of the low-energy region.

are generated in the focus of the optical beam (squares). For comparison, data obtained by the external-field method (dots) are shown as well.

Cook et al. initially attributed the $\mathrm{THz}$-generation process to four-wave rectification mediated by the third-order-nonlinearity of air [6] but later reported indications for a plasma-driven process [7]. In this four-wave-mixing picture, the nonlinear polarization in the focal region and respectively the THz field amplitude are given by

$$
E_{\mathrm{THz}} \propto P_{\text {focus }} \propto \chi^{(3)} E_{\omega}^{2} E_{2 \omega} .
$$

Here, $E_{\omega}$ and $E_{2 \omega}$ denote the optical field amplitudes of the fundamental and the SH pulses. As $E_{2 \omega}$ scales with the square of $E_{\omega}$ due to the SHG process in the $\mathrm{BBO}$ crystal, the $\mathrm{THz}$ field amplitude scales with the square of the laser pulse energy. This is indeed observed in the range of laser pulse energies from 100 to $250 \mu \mathrm{J}$.

At laser pulse energies below $100 \mu \mathrm{J}$, the measured amplitudes lie below the fit curve, and the amplitude values become unmeasurably small below $35 \mu \mathrm{J}$. This energy value corresponds to an intensity of about $1.5 \times 10^{14} \mathrm{~W} / \mathrm{cm}^{2}$, the ionization threshold of air [13]. In the experiments with a static bias applied to the focal region, we also find a threshold behaviour and attribute it to the onset of plasma formation $[5,8,9,12]$. Figure 5 (circles) shows that the amplitude of the external-bias THz signal scales linearly with the laser pulse energy. The linear fit curve to the data cuts the energy axis at the same value as in the case of SH biasing. This is a clear indication that in both cases plasma formation is a prerequisite for the generation of the observed $\mathrm{THz}$ radiation.

For the $\mathrm{THz}$ signal obtained with $\mathrm{SH}$ bias, we note that the amplitude of the $\mathrm{THz}$ transients falls below the fitted quadratic curve for pulse energies greater 
than $250 \mu \mathrm{J}$. At these high pulse energies, defocusing of the laser beam by the plasma [1] becomes important and limits the peak intensity of the laser pulse. In addition, at larger plasma volumes, phase mismatch between the optical and the $\mathrm{THz}$ waves and THz-absorption effects are likely to become significant.

When the dependence of the $\mathrm{THz}$ power on the pump power above the threshold is described by four-wave mixing, the value of $\chi^{(3)}$ is neither that of air nor that of the air plasma (so far no $\chi^{(3)}$ values have been reported for the plasma medium which exceed the value of $\chi^{(3)}$ of air [14]). A much larger value is needed in order to account for the measured data. Its origin lies in the generation process of the plasma. We have developed a microscopic model which is based on the non-linear effects introduced by the ionization process. The basic concept is as follows. The ionization of a molecule occurs at a certain electric field, which in our case is that of the optical waves. Because of the temporal and spatial asymmetry of the combined electric field of fundamental and second-harmonic waves, all charge carriers move into the same direction upon ionization. This leads to a polarization of the plasma, and to a plasma wave which is strongly damped, and hence to emission of a broad-band electromagnetic pulse. A detailed analysis which also takes into account the spatio-temporal evolution of the plasma will be published elsewhere [15].

\section{Comparison of the efficiency of $\mathrm{THz}$ sources}

If one compares $\mathrm{THz}$ emitters, firstly the question arises which criteria are appropriate to be compared. One may take into regard spectral properties, $\mathrm{THz}$ pulse energies, the maximum electric THz field, or signal-to-noise properties. For the plasma emitters considered in this paper the spectral properties do not differ significantly in the experimentally covered range of up to $3 \mathrm{THz}$. This also implies that the scaling factors between the THz-pulse energy and the square of the amplitude of the electric radiation field are nearly the same for all emitters. In the following, we will therefore compare only the electric field amplitudes.

Data for a standard large-area GaAs emitter are also included in Fig. 6 as state-of-the-art reference. It is important to point out that the highest $\mathrm{THz}$ field amplitudes (150 kV/cm, see star in Fig. 6) reached so far [10] have been obtained with pulsed bias fields. This method has the disadvantage that it creates large background noise. The higher signal level does not allow for an increased signal-to-noise value in measurements. With a static bias field of $1 \mathrm{kV} / \mathrm{cm}$, THz field amplitudes of up to $10 \mathrm{kV} / \mathrm{cm}$ can be reached as shown by the dots and the dotted line in Fig. 6. The noise properties are much better with static bias than with pulsed bias although one still has to deal with considerable noise because of the periodic recharging of the GaAs emitter. One characteristic feature of the GaAs emitter is its strong saturation at laser pulse energies of around $100 \mu \mathrm{J}$. If one wants to make use of the high laser pulse energies available with low-repetition-rate table-top laser systems (e.g., energies in the 10-100 mJ range are available 


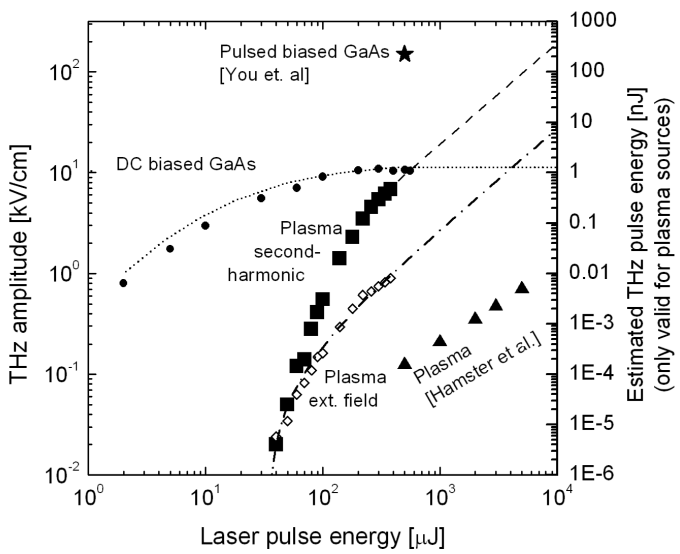

Fig. 6. Maximal electric field amplitude reached with the following emitters: large-area GaAs emitter with pulsed bias at $10.7 \mathrm{kV} / \mathrm{cm}(\star$; value taken from [10]) and with static bias at $1 \mathrm{kV} / \mathrm{cm}$ (•; values measured by us); plasma emitter with SH bias (()); plasma emitter with external field bias at $40 \mathrm{kV} / \mathrm{cm}(\diamond)$; plasma emitter based on ponderomotive forces (full $\triangle$, values extracted from the data of [4]). Data from plasma sources are linearly extrapolated. Data of the GaAs emitters are fitted with the saturation formula of [16]. THz pulse energies are estimated for the plasma sources based on experimentally determined conversion factors for emitters with comparable spectral properties.

with $10 \mathrm{~Hz}$ laser systems), it is necessary to employ emitters with larger area or a mosaic of emitters in order to increase the $\mathrm{THz}$ pulse energy. It is also interesting to point out that the spectrum of the GaAs emitter is shifted towards lower frequencies compared to the plasma sources. This leads to a lower $\mathrm{THz}$ field amplitude of a GaAs emitter compared to a plasma emitter with the same $\mathrm{THz}$ pulse energy.

We now come to the measured $\mathrm{THz}$ field amplitudes of the various plasma sources discussed in this paper. The data of the plasma sources have in common that they show a threshold behaviour at $35 \mu \mathrm{J}$ because of the onset of plasma formation at this laser energy value. The THz field amplitudes reached with both the ponderomotive-forces method and the external-bias method scale approximately linearly with the laser pulse energy. The $\mathrm{THz}$ pulse energy, respectively, scales with the square of the laser pulse energy. These dependencies are mainly related to the slightly superlinear increase in the plasma volume [15], which is partially compensated by phase mismatch and absorption effects occurring at larger plasma volumes.

Comparing the amplitudes of the THz fields generated at a laser pulse energy of $500 \mu \mathrm{J}$, one finds that the values for the ponderomotive-forces method and those for the external-field method are two, respectively one, order of magnitude below that of the statically biased GaAs emitter. The THz signal generated with the SH bias method is of about the same strength as that of the GaAs emitter. 
At lower laser pulse energies, in the range below $100 \mu \mathrm{J}$, the THz-generation efficiency of the SH bias method is comparable to that of the external-field method. At higher laser pulse energies, however, the SH bias approach offers the advantage of a stronger (quadratic) increase in the $\mathrm{THz}$ signal with laser pulse energy. An explanation for the stronger energy dependence is given below.

With respect to the noise properties, the plasma sources are far superior to the GaAs emitters. As pointed out above, the main source for noise is the current associated with the recharging of the GaAs emitter. In the case of the plasma emitters, recharging is either not required (SH bias method and ponderomotive-forces emitter) or of minor significance (external-field method).

Because of the strong saturation of the standard GaAs emitter, it is very interesting to discuss the potential of the plasma sources for operation in conjunction with very intense laser pulses with energies of $10 \mathrm{~mJ}$ and more. From the generation mechanism, one expects that for both the ponderomotive-forces method and the external-field method the $\mathrm{THz}$ signal will be proportional to the plasma volume, although partially compensated by phase mismatch and absorption effects. Indeed, the data of Hamster et al. [2-4] exhibit a linear increase in the $\mathrm{THz}$ field amplitude obtained by the external-field method at laser pulse energies up to $10 \mathrm{~mJ}$ (see Fig. 6). The situation is more difficult to analyze in the case of the SH bias method because of the SHG process involved. The maximum relative part of the laser pulse energy which can be converted to the SH signal is in the order of $15 \%$. This value results from the finite thickness of the BBO crystal and the maximum energy flux which can be applied to the crystal without damaging it. For our experimental setup this limit is reached at the maximally available pulse energy of $500 \mu \mathrm{J}$. If the laser pulse energy could be raised further, an increase in the $\mathrm{THz}$ amplitude would only result from the increase in the plasma volume provided that the ratio of $\mathrm{SH}$ to fundamental power is kept fixed at the maximum value of $15 \%$ (which would require to continuously enlarge the illuminated area of the BBO crystal). In this energy regime, we therefore expect only a linear increase in the $\mathrm{THz}$ signal amplitude as for the other plasma emitters. Under these assumptions, the SH-biased plasma emitter will produce THz-field amplitudes of the order of $150 \mathrm{kV} / \mathrm{cm}$ for pump pulse energies of $10 \mathrm{~mJ}$. This is equivalent to the very best values of GaAs emitters at the advantage of a broader spectrum and a much lower background noise.

Currently we are performing experiments at the front end of the PHELIX laser system at GSI (Gesellschaft für Schwerionenforschung, Darmstadt, Germany) in order to explore this laser pulse regime experimentally.

\section{Summary}

In summary, we have reviewed THz-pulse generation in laser-induced air plasmas. The emitter based on second-harmonic biasing emerges as an interesting 
source for $\mathrm{THz}$ applications. Its potential rises with the available laser pulse energy, and it should be explored further especially with regard to laser pulse energies at $1 \mathrm{~mJ}$ and above where alternative sources suffer from saturation effects.

\section{Acknowledgment}

This work was funded in part by GSI (Gesellschaft für Schwerionenforschung), Darmstadt, Germany, under contract no. OF-ROS.

\section{References}

[1] M. Mlejnek, E.M. Wright, J.V. Moloney, Phys. Rev. E 58, 4903 (1998).

[2] H. Hamster, A. Sullivan, S. Gordon, W. White, R.W. Falcone, Phys. Rev. Lett. 71, 2715 (1993).

[3] H. Hamster, A. Sullivan, S. Gordon, R.W. Falcone, Phys. Rev. E 49, 671 (1994).

[4] H. Hamster, Ph.D. thesis, University of Berkeley, 1993.

[5] T. Löffler, F. Jacob, H.G. Roskos, Appl. Phys. Lett. 77, 453 (2000).

[6] D.J. Cook, R.M. Hochstrasser, Opt. Lett. 25, 1210 (2000).

[7] D.J. Cook, J.-X. Chen, R.M. Hochstrasser, in: Ultrafast Phenomena XII, Springer Series, Springer, 2000, p. 197.

[8] T. Löffler, S. Eden, M. Thomson, H.G. Roskos, in: Ultrafast Phenomena XIII, Springer Verlag, 2002, p. 274.

[9] M. Kress, T. Löffler, S. Eden, M. Thomson, H.G. Roskos, Opt. Lett. 29, 1120 (2004).

[10] D. You, R.R. Jones, P.H. Bucksbaum, Opt. Lett. 18, 290 (1993).

[11] M. Geissler, T. Brabec, in: 28th EPS Conf. on Contr. Fusion and Plasma Phys., ECA, Vol. 25A, 2001, p. 2017.

[12] T. Löffler, H.G. Roskos, J. Appl. Phys. 91, 2611 (2002).

[13] S. Augst, D.D. Meyerhofer, D. Strickland, S.L. Chin, J. Opt. Soc. Am. B 8, 858 (1991).

[14] H. Harberland, M. Bonitz, H. Kremp, Phys. Rev. E 64, 026405 (2001).

[15] T. Löffler, Ph.D. thesis, JWG University Frankfurt Germany, 2003 (http://deposit.ddb.de/cgi-bin/dokserv?idn=96998541x).

[16] J.T. Darrow, X.-C. Zhang, D.H. Auston, IEEE J. Quantum Electron. 28, 2424 (1996). 